Check for updates

Cite this: RSC Adv., 2018, 8, 7351

Received 17th January 2018

Accepted 26th January 2018

DOI: $10.1039 / c 8 \mathrm{ra00503f}$

rsc.li/rsc-advances

\section{Fabrication of a magnetite/diazonium functionalized-reduced graphene oxide hybrid as an easily regenerated adsorbent for efficient removal of chlorophenols from aqueous solution $\dagger$}

\begin{abstract}
Xiaoqin Shen, ${ }^{a}$ Xiaolei Chen, ${ }^{b}$ Dejun Sun, ${ }^{b}$ Tao $W^{* b}$ and Yujiang Li (DD *a
A magnetic hybrid nanomaterial, which contains magnetite $\left(\mathrm{Fe}_{3} \mathrm{O}_{4}\right)$ particles and diazonium functionalizedreduced graphene oxide (DF-RGO), was fabricated via a three-pot reaction. First, the reduced graphene oxide (RGO) was synthesized via a redox reaction. Second, diazonium functionalized-RGO was prepared via a feasible chemical reaction. Third, $\mathrm{Fe}_{3} \mathrm{O}_{4}$ particles were loaded onto the surface of DF-RGO by covalent bonding, fabricating the M-DF-RGO hybrid. The fabricated hybrid was characterized by SEM, TEM, AFM, XRD, XPS, FT-IR, TGA, Raman spectroscopy, and magnetometry. The resulting M-DF-RGO hybrid possessed unique magnetic properties and was applied to remove 4-chlorophenol (4-CP) and 2,4-dichlorophenol (2,4-DCP) from aqueous solution. The adsorption of 4-CP and 2,4-DCP on the MDF-RGO hybrid was performed under various conditions, with respect to initial chlorophenol concentration, $\mathrm{pH}$, and contact time. The results suggest that the adsorption of 4-CP and 2,4-DCP onto the M-DF-RGO hybrid is strongly dependent on $\mathrm{pH}$ and weakly dependent on contact time. In addition, the adsorption isotherm of 4-CP and 2,4-DCP on the M-DF-RGO hybrid fits the Freundlich model well and the adsorption capacities of 4-CP and 2,4-DCP on M-DF-RGO reached 55.09 and $127.33 \mathrm{mg} \mathrm{g}^{-1}$, respectively, at $\mathrm{pH} 6$ and $25^{\circ} \mathrm{C}$. In this situation, intermolecular interactions including $\pi-\pi$ interactions and hydrogen bonding are operative. The calculated results of density functional theory further demonstrate that 2,4-DCP molecules could be more easily absorbed than 4-CP molecules by the $M$ DF-RGO hybrid. Moreover, the M-DF-RGO hybrid could be easily separated by a magnetic separation process, and showed good recyclability of more than five cycles.
\end{abstract}

\section{Introduction}

Chlorophenols are important industrial and chemical raw materials widely used in the plastics, cosmetics, polymers, paints/dyes, pharmaceutical and pesticide industries. ${ }^{\mathbf{1 - 4}}$ They are slightly acidic, appreciably soluble in water, and highly toxic to animals and human beings even at very low concentrations, and thus are considered one of the most major pollutants in wastewaters. ${ }^{5}$ Such wastewaters that contain chlorophenols are of increasing concern and represent a great potential hazard to both the environment and human health. Although chlorophenols can be biodegraded in the activated sludge process, the biodegradation rate of chlorophenols or phenolic

${ }^{a}$ Shandong Provincial Research Center for Water Pollution Control, School of Environmental Science \& Engineering, Shandong University, Jinan, 250100, PR China. E-mail: yujiang@sdu.edu.cn; Fax: +86-531-88363358; Tel: +86-531-88363358 ${ }^{b}$ Key Laboratory of Colloid \& Interface Science of Education Ministry, Shandong University, Jinan, 250100, PR China. E-mail: wutao@sdu.edu.cn; Fax: +86-53188365437; Tel: +86-531-88365437

$\dagger$ Electronic supplementary information (ESI) available. See DOI: 10.1039/c8ra00503f compounds is very low. Furthermore, the presence of chlorophenols in wastewaters can increase the susceptibility of a biological system to becoming disturbed. ${ }^{1-5}$ Hence, chlorophenols or phenolic compounds must be removed from wastewater before it is discharged into receiving water bodies or the environment.

To date, various techniques such as biological degradation, chemical oxidation, photodegradation, UV/ozone oxidation, and adsorption have been researched for the treatment of phenolic wastewater at high concentration. ${ }^{6-8}$ Of all of the known technologies, adsorption is considered to be the most effective, simplest, lowest-costing, and most frequently used method for the removal of chlorophenols or phenolic compounds. Various adsorbent materials such as clay minerals, metal hydroxides, resin, activated carbon and polymeric adsorbents have been applied for the removal of chlorophenols or phenolic compounds from aqueous solution. ${ }^{9-12}$ However, these traditional adsorbent materials still have certain problems that limit their practical applications, such as low selectivity, low adsorption capacity, poor dispersion stability, and difficulty in regeneration of the adsorbent material. As a result, 
the design and fabrication of new adsorbent materials to solve these problems are of significant importance.

Graphene is an excellent two-dimensional (2D) material, which possesses structural units that are similar to benzene rings that connect to each other, forming a 2D planar structure. ${ }^{13}$ Its attractive properties include high surface area, high electron mobility at room temperature, tunable optical properties, good thermal conductivity, and excellent mechanical strength. ${ }^{\mathbf{1 3 , 1 4}}$ As a result, graphene is applied in diverse fields, such as nanoelectronics, optical ultrasensitive sensors, fieldeffect transistors, and energy storage and conversion. ${ }^{\mathbf{1 4}-17}$ However, graphene has poor dispersivity and process stability. Graphene sheets tend to aggregate in the liquid phase due to van der Waals forces between graphene sheets, and this aggregation restricts the applications. Hence, covalent modification of graphene has been used to accomplish improvements of the physicochemical properties of graphene. Previous studies have demonstrated that graphene can be covalently functionalized by various direct or indirect functionalization methods. ${ }^{16-20}$ Either directly or indirectly covalently functionalized graphene is generated from graphene oxide (GO) as the precursor. GO, one of the most important derivatives of graphene, has a variety of oxygen-containing functional groups, such as hydroxyl, carbonyl, epoxide, and carboxyl groups, on its basal planes and edges. Taking into account these abundant oxygen-containing functional groups, GO can not only be well dispersed in water to form a stable colloidal dispersion, but also can potentially be used as nano-scale $2 \mathrm{D}$ building blocks for the fabrication of graphene-based composites. ${ }^{21,22}$ Among these oxygen-containing functional groups, the carboxyl groups are mainly located at the edges of the graphene sheets, and are excellent precursors for functionalizing various graphene-based composites without distorting the graphene plane. ${ }^{23}$ Therefore, the carboxyl group plays an important role in fabricating various new composites. However, other oxygen-containing functional groups (e.g. epoxide and carbonyl groups) on the planar surface of GO may disturb the covalent modification process. In this case, GO can be selectively reduced by thiourea dioxide, leaving the desired carboxyl groups and removing the undesired epoxide and carbonyl groups. This type of selectively reduced graphene is called carboxyl graphene or reduced graphene oxide (RGO). RGO can be used as a precursor and reacts with the functional groups of various organic molecules to introduce new functional groups for designing and fabricating new adsorbent materials. To the best of our knowledge, a magnetic diazonium functionalized-reduced graphene oxide (M-DF-RGO) hybrid has not been synthesized and applied in the removal of 4-CP and 2,4-DCP from aqueous solution.

In this work, the M-DF-RGO hybrid was synthesized via a three-pot reaction and used as an adsorbent to remove 4-CP and 2,4-DCP from aqueous solution. The fabricated M-DF-RGO hybrid was characterized using scanning electron microscopy, transmission electron microscopy, atomic force microscopy, $\mathrm{X}$ ray diffraction, X-ray photoelectron spectroscopy, Fourier transform infrared spectroscopy, thermogravimetric analysis, Raman spectroscopy, and magnetometry. 4-CP and 2,4-DCP were chosen as model pollutants to describe the adsorption behavior of chlorophenol molecules on the M-DF-RGO hybrid, and to elucidate the interactions between chlorophenol molecules and the M-DF-RGO hybrid. The effects of initial chlorophenol concentration, $\mathrm{pH}$, and contact time on the adsorption capacity were investigated. At the same time, the interactions between chlorophenol molecules and the M-DF-RGO hybrid were also analyzed using density functional theory. Finally, the reusability of the M-DF-RGO hybrid for 4-CP and 2,4-DCP was explored.

\section{Materials and methods}

\subsection{Materials}

Graphite powder $(<45 \mu \mathrm{m}$, $\geq 99.99 \%)$ was purchased from Sigma-Aldrich Co., Ltd. (USA). Thiourea dioxide (TUD, 99\%), hydrochloric acid ( $\mathrm{HCl}, 36-38 \%)$, sodium nitrite $\left(\mathrm{NaNO}_{2}, 98 \%\right)$, ferric chloride hexahydrate $\left(\mathrm{FeCl}_{3} \cdot 6 \mathrm{H}_{2} \mathrm{O}, \geq 98 \%\right)$, ferrous sulfate heptahydrate $\left(\mathrm{FeSO}_{4} \cdot 7 \mathrm{H}_{2} \mathrm{O}, \geq 99 \%\right)$, 4-chlorophenol (4CP, >99\%), 2,4-dichlorophenol (2,4-DCP, >99\%), 4-aminobenzoic acid (99\%), and sodium hydroxide $(\mathrm{NaOH}, 96 \%)$ were purchased from Sinopharm Chemical Reagent Co., Ltd. (Shanghai, China). All of the other chemicals were analytically pure, and used without further purification. Milli-Q water (Millipore, USA) was used throughout the experimental process.

\subsection{Preparation of GO and RGO}

2.2.1 Preparation of GO. GO was first prepared from natural graphite powder according to Hummers' method with some modification. ${ }^{24}$ In brief, graphite $(1 \mathrm{~g})$, sodium nitrate $\left(\mathrm{NaNO}_{3}, 1 \mathrm{~g}\right)$, and concentrated sulfuric acid $\left(\mathrm{H}_{2} \mathrm{SO}_{4}, 23 \mathrm{~mL}\right)$ were mixed and stirred in a three-neck flask in an ice bath. Potassium permanganate $\left(\mathrm{KMnO}_{4}, 3 \mathrm{~g}\right)$ was then slowly added with continuous vigorous stirring and cooling to keep the temperature below $20{ }^{\circ} \mathrm{C}$. The ice bath was removed and replaced by an oil bath, and the system was heated to $35^{\circ} \mathrm{C}$ and stirred for $30 \mathrm{~min}$, at which time Milli-Q water was added slowly, producing a large exotherm to $98{ }^{\circ} \mathrm{C}$. The reaction was kept at $98{ }^{\circ} \mathrm{C}$ for $15 \mathrm{~min}$. Then, the reaction was cooled using a water bath for $10 \mathrm{~min}$. More Milli-Q water $(400 \mathrm{~mL})$ and hydrogen peroxide solution $\left(\mathrm{H}_{2} \mathrm{O}_{2}, 30 \%, 10 \mathrm{~mL}\right)$ were added, producing another exotherm. After air cooling, the mixtures were separated using centrifugation at $8000 \mathrm{rpm}$, and washed initially with $5 \% \mathrm{HCl}$ until $\mathrm{SO}_{4}{ }^{2-}$ was no longer detectable with barium chloride $\left(\mathrm{BaCl}_{2}\right)$. The mixtures were then washed several times with acetone and dried in a vacuum drier at $60{ }^{\circ} \mathrm{C}$ for $12 \mathrm{~h}$ in order to obtain graphite oxide. The GO dispersion was obtained after sonication of graphite oxide for $2 \mathrm{~h}$ in Milli-Q water.

2.2.2 Preparation of RGO. RGO was obtained from the reaction of thiourea dioxide with GO. ${ }^{14}$ In brief, graphite oxide powder $(200 \mathrm{mg})$ was dispersed into Milli-Q water $(200 \mathrm{~mL})$ under ultrasonication to produce a homogeneous brownish yellow GO dispersion. After the $\mathrm{pH}$ of the GO dispersion was adjusted to 10 by the addition of ammonia water $\left(\mathrm{NH}_{3} \cdot \mathrm{H}_{2} \mathrm{O}\right.$, $30 \%)$, thiourea dioxide $(0.2 \mathrm{~g})$ was added to the $\mathrm{GO}$ dispersion, and the mixture was stirred for $30 \mathrm{~min}$ at $40{ }^{\circ} \mathrm{C}$ under nitrogen protection. Until the reduction was completed, several drops of 
dilute $\mathrm{HCl}(0.5 \%, \mathrm{w} / \mathrm{v})$ were added dropwise to the as-prepared RGO dispersion in order to cause the ammonia water to form a kind of inorganic salt that could be washed away with Milli-Q water when it was filtered. The black RGO product was finally obtained after the filtered residue was freeze-dried for $24 \mathrm{~h}$.

\subsection{Preparation of DF-RGO}

The chemically functionalized RGO was prepared by covalently attaching organic groups on the planar surface of RGO. The aryl diazonium salt solution was first prepared according to the following method: $4.1 \mathrm{mmol} \mathrm{L}^{-1} \mathrm{NaNO}_{2}$ and $1.2 \mathrm{mmol} \mathrm{L}{ }^{-1} 4$ aminobenzoic acid were dissolved in $15 \mathrm{~mL}$ dilute $\mathrm{NaOH}$ solution $(0.25 \%, \mathrm{w} / \mathrm{v})$. The as-prepared solution was then added slowly to $0.1 \mathrm{~mol} \mathrm{~L}^{-1} \mathrm{HCl}$ solution $(20 \mathrm{~mL})$ in an ice bath with continuous mechanical stirring.

Before the aryl diazonium salt solution was added into the RGO colloidal dispersion, the $\mathrm{pH}$ value of the RGO colloidal dispersion was adjusted to 6 by the addition of dilute $\mathrm{HCl}$ solution in an ice bath. During the adjustment of the $\mathrm{pH}$ value from basic to acidic, the RGO colloidal dispersion $\left(1 \mathrm{~g} \mathrm{~L}^{-1}\right)$ precipitated and produced brown flocculent precipitates. Under continuous stirring, the aryl diazonium salt solution was immediately added dropwise to the RGO flocculent precipitates. Then, the mixture was kept in the ice bath without stirring for $2 \mathrm{~h}$ in order to adsorb sufficient aryl diazonium ions onto the RGO sheets. After $2 \mathrm{~h}$, the mixture was heated to $80^{\circ} \mathrm{C}$ for $10 \mathrm{~h}$, and then cooled to room temperature. A homogeneous black dispersion of diazonium salt functionalized-RGO sheets was obtained. The resulting product was filtered and washed with Milli-Q water and ethanol. Finally, the DF-RGO sample was obtained by freeze-drying for $24 \mathrm{~h}$.

\subsection{Preparation of M-DF-RGO hybrid}

To prepare the M-DF-RGO hybrid, DF-RGO (0.4 g) was first ultrasonicated in $200 \mathrm{~mL}$ Milli-Q water to form a homogeneous colloidal dispersion. Then, the colloidal dispersion was transferred to a three-neck flask (equipped with a reflux condenser) under a nitrogen atmosphere. A mixed aqueous solution of $\mathrm{FeCl}_{3} \cdot 6 \mathrm{H}_{2} \mathrm{O}(0.5597 \mathrm{~g})$ and $\mathrm{FeSO}_{4} \cdot 7 \mathrm{H}_{2} \mathrm{O}(0.5592 \mathrm{~g})$ in $20 \mathrm{~mL}$ Milli-Q water was added to the DF-RGO colloidal dispersion. The mixture was then heated to $80{ }^{\circ} \mathrm{C}$ and stirred under nitrogen protection. After that, $250 \mathrm{~mL} \mathrm{NH}{ }_{3} \cdot \mathrm{H}_{2} \mathrm{O}$ solution $(30 \%)$ was added to adjust the $\mathrm{pH}$ to 10 , and the mixtures were stirred and maintained at $80{ }^{\circ} \mathrm{C}$ for $30 \mathrm{~min}$. Finally, trisodium citrate $(0.1 \mathrm{~g})$ was added to the mixture and the temperature was raised to $95{ }^{\circ} \mathrm{C}$, resulting in a black suspension. The obtained product was separated with a permanent magnet, washed several times with Milli-Q water, and dried under vacuum at $60{ }^{\circ} \mathrm{C}$.

\subsection{Characterization}

The surface morphologies and structural features of GO, RGO, DF-RGO, and M-DF-RGO were characterized using a JEOL-2010 transmission electron microscope (TEM, JEOL, Japan) and a JSM-6330 F scanning electron microscope (SEM, JEOL, Japan). Atomic force microscopy images of the samples were acquired using a Multimode Nano 4 microscope system (AFM, Bruker,
USA) in the tapping mode. X-ray diffraction patterns of the samples were recorded by an X'Pert Super X-ray diffractometer (XRD, Philips, Holland) using $\mathrm{Cu} \mathrm{K} \alpha(\lambda=1.540598 \AA)$ irradiation at $40 \mathrm{kV}$ and $40 \mathrm{~mA}$ in the $2 \theta$ range of $5-70^{\circ}$ at a scanning rate of $0.02^{\circ} \mathrm{s}^{-1}$. Fourier transform infrared spectra of the samples were recorded using a Nicolet Nexus 670 spectrometer (FI-IR, Thermo Fisher Scientific, USA) using $\mathrm{KBr}$ pellets in the range of $400-4000 \mathrm{~cm}^{-1}$, with a resolution of $2 \mathrm{~cm}^{-1}$. X-ray photoelectron spectroscopy was performed using a XSAM800 spectrometer (XPS, Kratos Company, UK). Raman spectra of the samples were obtained using a LabRAM electron energy spectrometer (HORIBA Company, France) with a $632.8 \mathrm{~nm} \mathrm{He}-\mathrm{Ne}$ excitation source. Thermogravimetric analysis was performed using TG209FI thermal analysis apparatus (TGA, NETZSCH, Germany) at a heating rate of $10{ }^{\circ} \mathrm{C} \mathrm{min}^{-1}$ from 40 to $700{ }^{\circ} \mathrm{C}$. Magnetic curves of the samples were measured using a 7410 vibrating sample magnetometer (VSM, Cryotronics Inc. United States, USA) at room temperature.

\subsection{Adsorption experiments and recycling tests}

Batch experiments were performed to test the removal efficiency of 4-CP and 2,4-DCP from aqueous solution using the M-DFRGO hybrid. The effects of initial chlorophenol concentration, $\mathrm{pH}$, and contact time on the removal efficiency of 4-CP and 2,4DCP were investigated. An initial experiment was conducted to determine the amount of adsorbent required. According to this experiment, $0.01 \mathrm{~g}$ of adsorbent was added to $10 \mathrm{~mL}$ phenolic aqueous solution. The initial $\mathrm{pH}$ of the aqueous solution was adjusted from 3 to 11 by adding either dilute $\mathrm{HCl}\left(0.1 \mathrm{~mol} \mathrm{~L}^{-1}\right)$ or dilute $\mathrm{NaOH}\left(0.1 \mathrm{~mol} \mathrm{\textrm {L } ^ { - 1 }}\right)$. The initial chlorophenol concentration was in the range of $10-300 \mathrm{mg} \mathrm{L}^{-1}\left(100 \mathrm{mg} \mathrm{L}^{-1}\right.$ for the adsorption study and $10-300 \mathrm{mg} \mathrm{L}^{-1}$ for the isotherm studies). The contact time ranged from 0 to $30 \mathrm{~min}$. The mixtures were continuously shaken on a mechanical shaker (SHZ-82, Jintan, China) for $30 \mathrm{~min}$ at $298 \mathrm{~K}$. After $30 \mathrm{~min}$, the mixtures were easily separated by a magnetic separation process, and the concentration of the residual 4-CP and 2,4-DCP was analyzed using a UV-vis adsorption spectrometer (UV-1601, Shimadzu, Japan) at wavelengths of 279 and $286 \mathrm{~nm}$ for 4-CP and 2,4-DCP, respectively.

Recycling tests were carried out to assess the recyclability of the M-DF-RGO hybrid. After adsorption experiments, the adsorbent particles were collected using a magnet. The 4-CPand 2,4-DCP-adsorbed adsorbent particles were washed five times with $0.01 \mathrm{~mol} \mathrm{~L}^{-1} \mathrm{NaOH}$ solution and ethanol, respectively, and vacuum-dried at $60{ }^{\circ} \mathrm{C}$ for $4 \mathrm{~h}$. The recovered adsorbent particles were again used for subsequent adsorption experiments. Five cycles were performed to evaluate the reusability of the M-DF-RGO hybrid.

\subsection{Computational models and methodology}

All of the geometrical structures were optimized using the Density Functional Theory (DFT) method with the PBE0 functional $^{25}$ in combination with the all-electron $6-31 G^{*}$ basis set. $^{26,27}$ Grimme-type dispersion corrections (D3) were included throughout $^{28}$ and the basis set superposition error (BSSE) was 
also considered for absorbing systems using the counterpoise method proposed by Boys and Bernardi. ${ }^{29}$ All calculations were carried out using the Gaussian09 package. ${ }^{30-32}$

Fig. S4† shows the molecular models of DF-RGO, and the added $p$-carboxyphenyl groups have been attached to the surface of the graphene sheets. It should be noted that in the conditions of our calculations, we intentionally neglect $\mathrm{Fe}_{3} \mathrm{O}_{4}$ particles, which did not work for the adsorption of chlorophenols (Fig. S5 $\dagger$ ). The adsorption energy $\left(E_{\text {ad }}\right)$ was calculated as $E_{\mathrm{ad}}=E_{\mathrm{G}}+E_{\mathrm{X}}-E_{\mathrm{G}-\mathrm{X}}$, where $E_{\mathrm{G}}$ corresponds to the total energies of DF-RGO; $E_{\mathrm{X} 1}$ corresponds to the total energies of the 4-CP system in isolated form; $E_{\mathrm{X} 2}$ corresponds to the total energies of the 2,4-DCP system in isolated form; $E_{\mathrm{G}-\mathrm{X} 1}$ is the total energy of the DF-RGO and 4-CP complex system; and $E_{\mathrm{G}-\mathrm{X} 2}$ is the total energy of the DF-RGO and 2,4-DCP complex system. A positive value of $E_{\mathrm{ad}}$ indicates that the adsorption is exothermic, and a higher positive value of $E_{\mathrm{ad}}$ corresponds to a stronger adsorption capacity of the adsorbate onto the DFRGO hybrid.

\section{Results and discussion}

\subsection{Characterization}

The morphologies and microstructures of GO, RGO, DF-RGO, and M-DF-RGO were studied using SEM and TEM techniques. As shown in Fig. 1a, the thin films of GO sheets with slight wrinkles and smooth surfaces were exfoliated. RGO (Fig. 1b) with a crumpled nano-sheet structure was transparent. The microstructure of DF-RGO (Fig. 1c) was similar to that of GO, which appeared transparent and was slightly folded. However, the contrast of DF-RGO was higher due to functionalization. As shown in Fig. 1d, magnetic nanoparticles were uniformly distributed and exhibited high-density coverage on the entire DF-RGO sheet surface, and no particle agglomerates were observed. The reason for this is that the diazonium functionalization of reduced graphene oxide significantly increased the number of carboxyl groups that can perfectly interact with $\mathrm{Fe}_{3} \mathrm{O}_{4}$ nanoparticles. From the SEM image of GO (Fig. 1e), it can be seen that the graphene sheets exhibit a wrinkled feature. Fig. 1f shows that RGO possesses a crumpled or wavy silk-like structure. Moreover, a flake-like structure can also be observed at the edge of the RGO sheets. From Fig. 1g, it can be seen that DF-RGO possesses a homogeneous and wrinkled structure. At the same time, crumpled wave-like carbon sheets can also be observed, which are characteristic of the single-layer graphene sheets. ${ }^{33}$ As shown in Fig. $1 \mathrm{~h}$, the $\mathrm{Fe}_{3} \mathrm{O}_{4}$ NPs appear as bright dots, and the large graphene flakes exhibit a slightly wrinkled surface.

AFM images confirmed that evaporated dispersions of GO, RGO, DF-RGO, and M-DF-RGO are composed of isolated graphitic sheets (Fig. S1a-d $\dagger$ ). On average, the thickness of the GO sheets is $\sim 0.90 \mathrm{~nm}$, while the thickness of RGO is $\sim 0.88 \mathrm{~nm}$, which implies that the oxygenated groups are partially reduced to re-establish the conjugated graphite network. Considering the intrinsic monolayer ripples owing to thermal fluctuations, it can be concluded that the RGO sheets are mixtures of monolayers and bilayers. The thickness of single layer DF-RGO is measured to be 1.1-1.5 nm, which is slightly higher than that of RGO. The addition of thickness may be attributed to the perpendicular covalent attachment of the functional group moieties. The M-DF-RGO hybrid was redispersed to form a metastable dispersion and drop-dried on a mica substrate for the AFM study. We found that even after a long period of sonication during the preparation of the AFM specimen, the magnetic nanoparticles are still strongly anchored on the surface of the DF-RGO sheets, suggesting a strong interaction between the magnetic nanoparticles and the DF-RGO sheets. The thickness of the M-DF-RGO sheets was in the range of 10$12 \mathrm{~nm}$. Since the size of the magnetic nanoparticles is about $10.12 \mathrm{~nm}$ (according to the XRD results) and the thickness of the DF-RGO is about 1.1-1.5 nm, it is reasonable to conclude that the hybrid consists of monolayer DF-RGO and magnetic nanoparticles. ${ }^{34}$

The powder X-ray diffraction (XRD) patterns are shown in Fig. 2a. Graphene oxide exhibited a diffraction peak at $2 \theta=$ $10.6^{\circ} .^{35}$ As calculated from the Bragg equation, the interlayer spacing of GO $(0.82 \mathrm{~nm})$ was enhanced after graphite $(0.34 \mathrm{~nm})$ was oxidized, which may be due to the introduction of oxygencontaining functional groups and the adsorption of water molecules. For RGO, a weak and broad diffraction peak (002) was observed at $2 \theta=22.8^{\circ}$, while the (001) diffraction peak of GO almost disappeared, which again proved that most of the
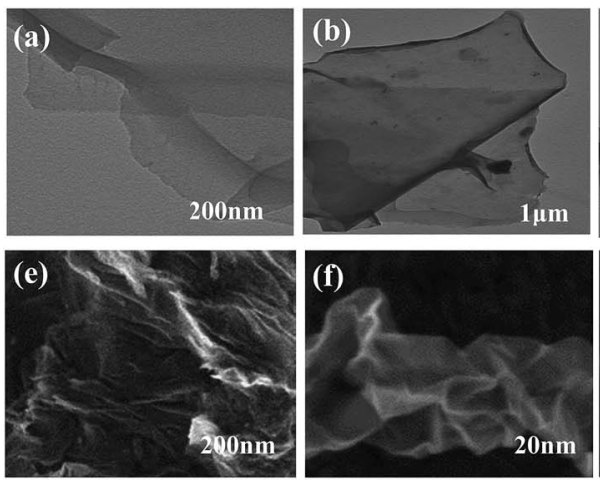
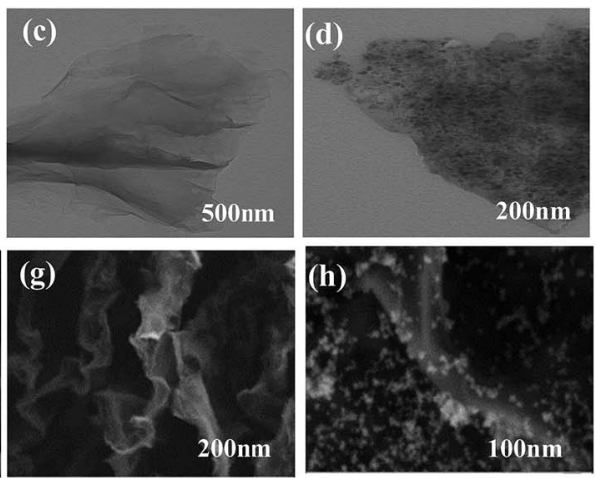

Fig. 1 TEM images of (a) GO, (b) RGO, (c) DF-RGO, and (d) M-DF-RGO; and SEM images of (e) GO, (f) RGO, (g) DF-RGO, and (h) M-DF-RGO. 
(a)

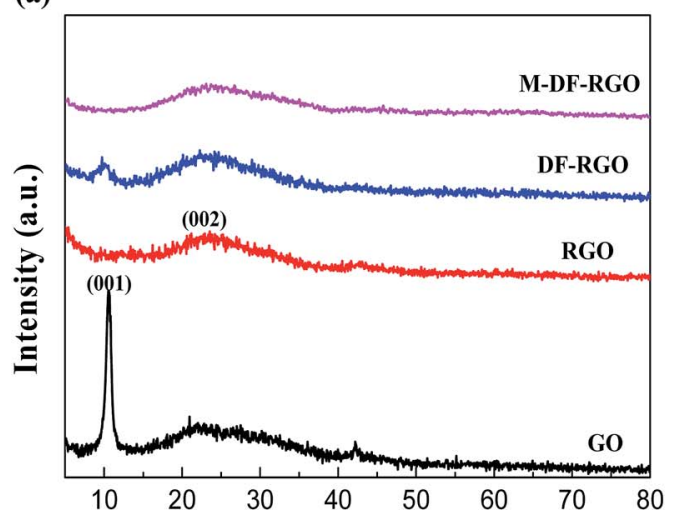

(c)

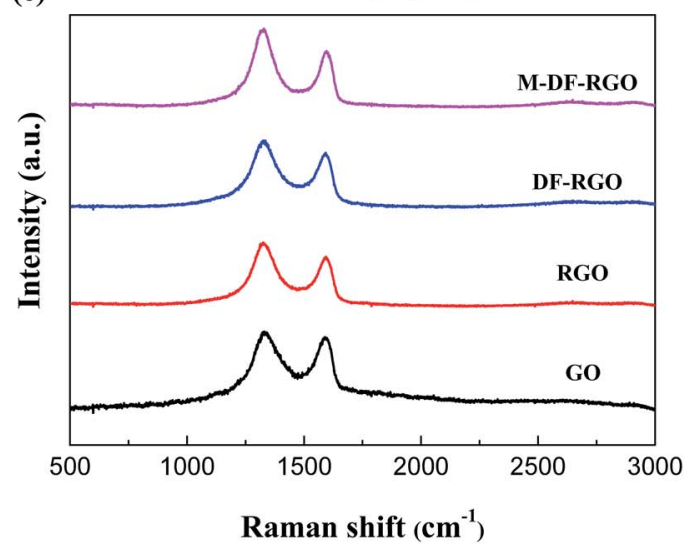

(b)

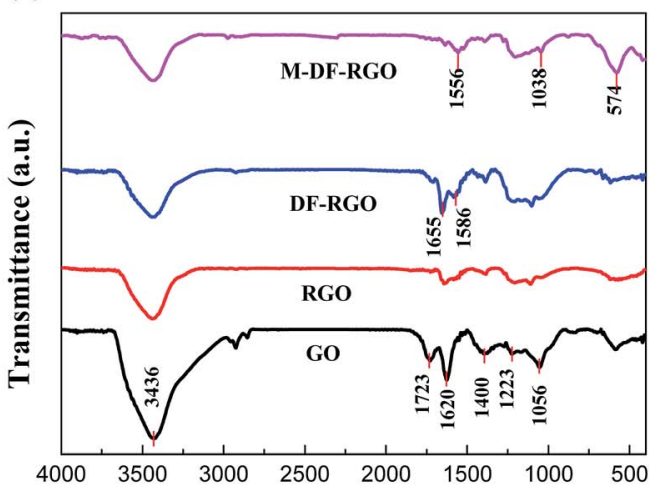

Wavenumbers $\left(\mathbf{c m}^{-1}\right)$

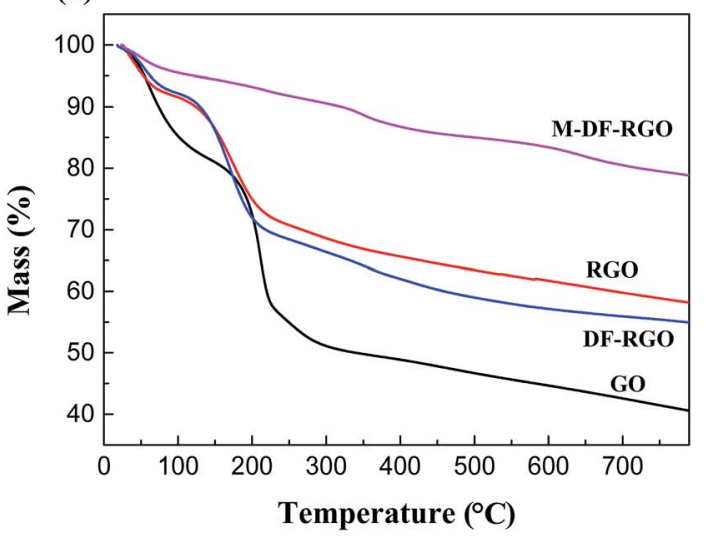

Fig. 2 Characterization of samples. (a) XRD spectra; (b) FTIR spectra; (c) Raman spectra; (d) TGA curves.

oxygen-containing functional groups on the surface of GO had been removed. Concerning DF-RGO, a broad reflection peak $\left(\sim 20^{\circ}\right)$ exists that is associated with the (002) RGO planes. The interlayer distance of DF-RGO $(0.90 \mathrm{~nm})$ is larger than that of graphene oxide $(0.82 \mathrm{~nm})$ as a result of the presence of $-\mathrm{C}_{6} \mathrm{H}_{5}-$ $\mathrm{COOH}$ groups on both sides of the RGO plane. The XRD pattern of M-DF-RGO exhibited weak reflections at $2 \theta=35.84^{\circ}$ (311), which is consistent with the existence of iron oxide either in $\mathrm{Fe}_{3} \mathrm{O}_{4}$ or $\gamma-\mathrm{Fe}_{2} \mathrm{O}_{3}$ phases. ${ }^{36}$ The adsorbed nanoparticles act as spacers that limit the restacking of graphene layers. ${ }^{37}$

The FTIR spectra of the samples are shown in Fig. $2 \mathrm{~b}$. The characteristic absorption peaks of graphite oxide at 1723, 1620, 1400,1223 , and $1056 \mathrm{~cm}^{-1}$ are attributed to the stretching vibration of $\mathrm{C}=\mathrm{O}, \mathrm{C}=\mathrm{C}, \mathrm{O}-\mathrm{H}, \mathrm{C}-\mathrm{O}-\mathrm{C}$, and $\mathrm{C}-\mathrm{O}$, respectively. An intense and broad peak at $\sim 3436 \mathrm{~cm}^{-1}$ could be assigned to the $\mathrm{O}-\mathrm{H}$ stretching vibration. ${ }^{38}$ For RGO, the absorption peaks of the $\mathrm{C}=\mathrm{O}, \mathrm{O}-\mathrm{H}, \mathrm{C}-\mathrm{O}$, and $\mathrm{C}-\mathrm{O}-\mathrm{C}$ groups decreased remarkably or even disappeared, while the peaks of the carbonyl groups were retained. The absorption peak at $794 \mathrm{~cm}^{-1}$ of DF-RGO is associated with stretching of the $\mathrm{C}-\mathrm{H}$ vibration of the benzoic acid group. ${ }^{39}$ Moreover, the peak at $1636 \mathrm{~cm}^{-1}$ can be assigned to the $\mathrm{C}=\mathrm{O}$ in a carboxylic acid and the intensity is evidently higher than that for bare GO, indicating that RGO has been successfully functionalized. The FTIR spectrum of M-DF-RGO differs from that of DF-RGO, as evidenced by the weakening of the $\mathrm{C}-\mathrm{O}$ peak at $3430 \mathrm{~cm}^{-1}$. This suggests that heat treatment in an alkaline medium removed oxygen from the surface of DFRGO. Two additional vibrational peaks appeared at approximately 1440 and $1380 \mathrm{~cm}^{-1}$. These derived from the formation of either a monodentate complex or a bidentate complex between the carboxyl group and Fe of the magnetic particles, indicating that the iron oxide nanoparticles were covalently bonded to the surface of DF-RGO by Fe-O-C bonds. ${ }^{36}$

Raman spectroscopy is a tool utilized in order to analyze ordered and disordered crystal structures of graphene based materials. Fig. 2c shows the Raman spectra of GO, RGO, DFRGO, and the M-DF-RGO hybrid. The ratio of intensities between the $\mathrm{D}$ and $\mathrm{G}$ bands $\left(I_{\mathrm{D}} / I_{\mathrm{G}}\right)$ of the samples in Raman spectroscopy is regarded as a measure of defects in samples. ${ }^{40}$ The ratio of the $\mathrm{D}$ to $\mathrm{G}$ band intensities $\left(I_{\mathrm{D}} / I_{\mathrm{G}}\right)$ increased from 1.19 to 1.44 after GO was reduced by TUD. Meanwhile, a general characteristic of the spectrum of RGO is the shift to a lower wavenumber, suggesting that the average size of the in-plane $\mathrm{sp}^{2}$ domain had decreased, while the number of $\mathrm{sp}^{2}$ carbon atoms increased for RGO. ${ }^{41}$ This means that the electronic conjugation of RGO was restored, which is consistent with the XRD results. The $I_{\mathrm{D}} / I_{\mathrm{G}}$ ratio for DF-RGO is approximately 1.52 , indicating that the aryl diazonium treatment led to a higher degree of disorder and defect bond formation, and some functional groups were grafted onto the surface of RGO. ${ }^{42}$ The D 
band of the M-DF-RGO composite is shifted downward by approximately $10 \mathrm{~cm}^{-1}$ compared to DF-RGO, while the $I_{\mathrm{D}} / I_{\mathrm{G}}$ ratio was calculated to be approximately 1.46 due to the reduction of DF-RGO during the co-precipitation reaction process. $^{43}$

The TGA curves of the samples are presented in Fig. 2d. For GO powders, significant weight loss at $100-150{ }^{\circ} \mathrm{C}$ and $150-$ $300{ }^{\circ} \mathrm{C}$ of $\sim 19.03 \mathrm{wt} \%$ and $\sim 30.87 \mathrm{wt} \%$, respectively, can be observed, which is attributed to the decomposition of oxygen functional groups and carbon skeleton oxidation, respectively. ${ }^{\mathbf{4 4}}$ In contrast, RGO possesses better thermal stability than GO, which is shown by a mass loss of just $\sim 15.22 \mathrm{wt} \%$ at $130-262{ }^{\circ} \mathrm{C}$ that arose from the residual carboxyl groups on the plane of RGO. TGA was employed to gauge the degree of functionalization of DF-RGO, and it enables comparison of the weight loss of the functionalized sample and RGO. The defunctionalization of DF-RGO is determined to occur at $200-700{ }^{\circ} \mathrm{C} .{ }^{45}$ In addition, MDF-RGO exhibited a lower thermal stability than DF-RGO. There was also a slow weight loss $(\sim 9.8 \mathrm{wt} \%)$ at a low temperature $\left(<100{ }^{\circ} \mathrm{C}\right)$, which can be attributed to the loss of the residual or absorbed solvent. The obvious weight loss occurring at and above $595{ }^{\circ} \mathrm{C}$ may be ascribed to the breakdown of the $-\mathrm{COO}$ group coordinated with $\mathrm{Fe}_{3} \mathrm{O}_{4}$ nanoparticles in the M-DF-RGO hybrid. ${ }^{46}$

The magnetization curves of $\mathrm{Fe}_{3} \mathrm{O}_{4}$ and M-DF-RGO were measured using VSM at room temperature, as shown in Fig. 3. The saturation magnetization of the M-DF-RGO hybrid was smaller than that of pure bulk $\mathrm{Fe}_{3} \mathrm{O}_{4}\left(79.20 \mathrm{emu} \mathrm{g}^{-1}\right)$ due to the smaller size of $\mathrm{Fe}_{3} \mathrm{O}_{4}$ nanoparticles and the existence of DFRGO. ${ }^{33}$ Meanwhile, the specific saturation magnetization of MDF-RGO is 28.46 emu $^{-1}$, which can be attributed to the relatively high amount of $\mathrm{Fe}_{3} \mathrm{O}_{4}$ nanoparticles loaded on DF-RGO. ${ }^{47}$

$\mathrm{X}$-ray photoelectron spectroscopy (XPS) is a sensitive analytical technique used to determine the surface elemental compositions and types of functional groups available on carbon materials. ${ }^{15,21,34}$ Table 1 lists the elementary compositions of the surfaces of the samples.

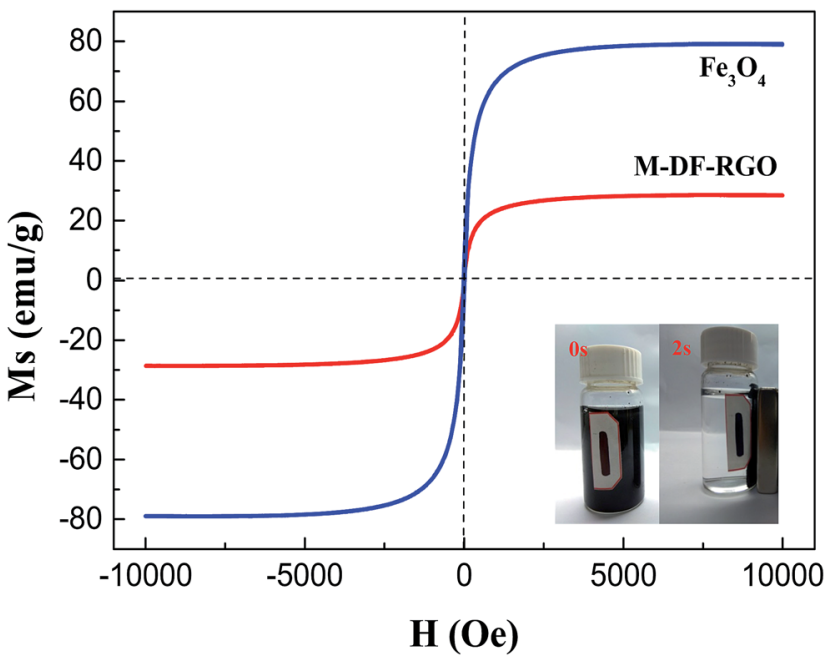

Fig. 3 The magnetization curves of the samples, with the inset showing the separation efficiencies of M-DF-RGO.
Table 1 Surface element analysis of GO, RGO, DF-RGO, and M-DF$\mathrm{RGO}$ (relative atomic percentage)

\begin{tabular}{lllll}
\hline Sample & C 1s & O 1s & $\begin{array}{l}\text { O 1s/C } \\
\text { 1s }\end{array}$ & Fe 2p \\
\hline GO & 63.88 & 36.12 & 0.56 & - \\
RGO & 78.00 & 36.12 & 0.28 & - \\
DF-RGO & 75.82 & 21.99 & 0.29 & - \\
M-DF-RGO & 64.50 & 26.98 & 0.42 & 8.52 \\
\hline
\end{tabular}

The high resolution XPS C 1s spectrum is shown in Fig. 4. For GO, the raw peaks can be divided into five individual peaks at $284.6 \mathrm{eV}(\mathrm{C}=\mathrm{C}), 285.6 \mathrm{eV}(\mathrm{C}-\mathrm{C}), 286.6 \mathrm{eV}(\mathrm{C}-\mathrm{O}), 287.4 \mathrm{eV}(\mathrm{C}=$ $\mathrm{O})$, and $288.6 \mathrm{eV}(\mathrm{O}-\mathrm{C}=\mathrm{O}) .{ }^{23,43}$ The high resolution XPS $\mathrm{C} 1 \mathrm{~s}$ spectrum of RGO showed a significant decrease of signals at $286-288 \mathrm{eV}$, which can be ascribed to the reduction of $\mathrm{C}-\mathrm{O} / \mathrm{C}-$ $\mathrm{O}-\mathrm{C}$ and $\mathrm{C}=\mathrm{O}$ functionalities. The relative atomic percentage of oxygen obviously decreased from $36 \%$ for GO to $21 \%$ for RGO, while the relative atomic percentage of $\mathrm{C}=\mathrm{C}$ groups on $\mathrm{RGO}$ increased to some extent in comparison with that on GO. However, the actual amount of the carboxyl functional groups on RGO remained largely the same. Upon reaction with aryl diazonium salts, the ratio of the $-\mathrm{COOH}$ peak area increased rapidly to $4.2 \%$ for DF-RGO from $2.0 \%$ for GO. All of these data proved that the surface of RGO had been covalently modified by p-carboxyphenyl groups through aryl diazonium treatment. Compared with DF-RGO, the relative content of $\mathrm{C}=\mathrm{C}$ groups on M-DF-RGO is higher, and the relative intensities of $\mathrm{C}-\mathrm{O} / \mathrm{C}-\mathrm{O}-\mathrm{C}$ and $\mathrm{C}=\mathrm{O}$ functionalities are lower, indicating the likelihood of DF-RGO to be reduced partially under alkaline conditions during the preparation of the M-DF-RGO hybrid. In the Fe 2p spectrum (Fig. S2†), the peaks at 711.8 and $725.1 \mathrm{eV}$ are attributed to $\mathrm{Fe} 2 \mathrm{p}_{3 / 2}$ and $\mathrm{Fe} 2 \mathrm{p}_{1 / 2}$. The spin-orbital splitting of the Fe $2 \mathrm{p}$ peak is broad due to a small chemical shift between $\mathrm{Fe}^{2+}$ and $\mathrm{Fe}^{3+},{ }^{47}$ both of which are present in $\mathrm{Fe}_{3} \mathrm{O}_{4}$.

\subsection{Chlorophenol removal studies}

3.2.1 Effect of $\mathbf{p H}$. The M-DF-RGO hybrid was used for the removal of chlorophenols (4-CP and 2,4-DCP) from aqueous solutions. The solution $\mathrm{pH}$ has a major influence on the adsorption behavior of the adsorbent. ${ }^{48}$ Specifically, the surface charge of the adsorbent and the dissociation state of the phenolic compounds in the solution were closely related to the solution pH. As shown in Fig. 5, the $q_{\mathrm{e}}$ values of both 4-CP and 2,4-DCP exhibited little change until $\mathrm{pH}=\mathrm{pKa}$ (9.38 and 7.89, respectively). ${ }^{49}$ As the $\mathrm{pH}$ continued to increase, the $q_{\mathrm{e}}$ values of the two chlorophenols rapidly decreased. The reason for this is that chlorophenol neutral molecules mostly dissociated to anions at $\mathrm{pH}>\mathrm{pKa}$, which would exhibit electrostatic repulsion with the negatively charged surface of the adsorbent according to the negative zeta potentials at high pH (Fig. S3†).

3.2.2 Adsorption kinetics. The adsorption kinetics of two chlorophenols on M-DF-RGO were measured at $298 \mathrm{~K}$, and the concentration of chlorophenols and adsorbent are $100 \mathrm{mg} \mathrm{L}^{-1}$ and $1.0 \mathrm{~g} \mathrm{~L}^{-1}$, respectively. As shown in Fig. 6, the adsorption rate of the chlorophenols rapidly increased at the beginning of 

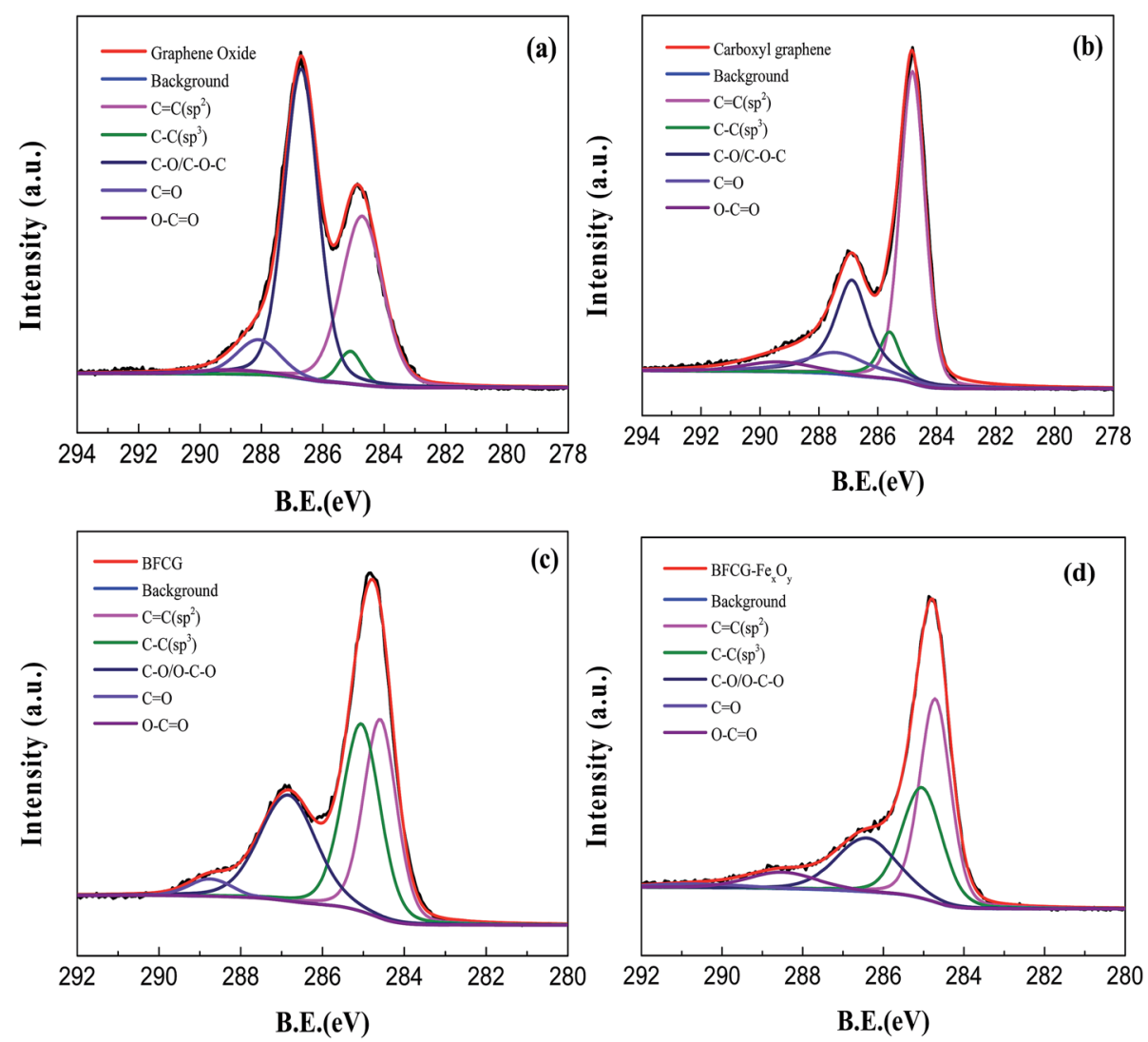

Fig. 4 High resolution C 1s core level XPS spectra of (a) GO, (b) RGO,

(c) DF-RGO, and (d) M-DF-RGO.

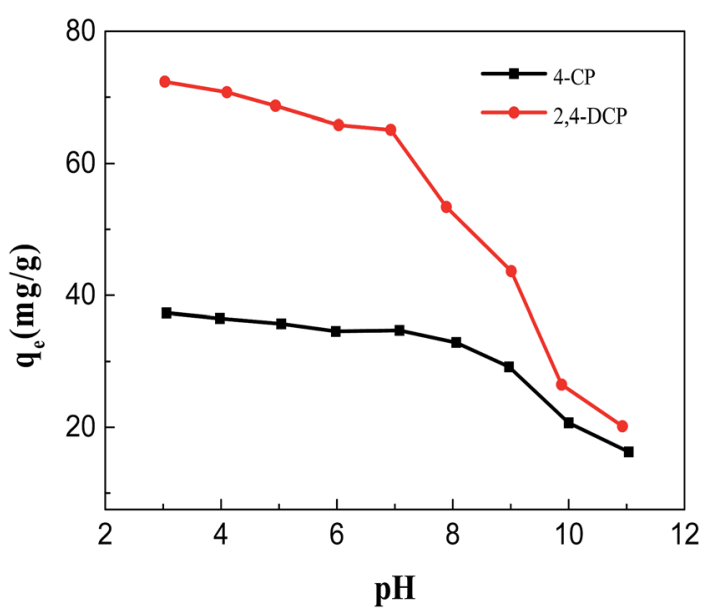

Fig. 5 The effect of $\mathrm{pH}$ on the removal efficiencies of 4-CP and 2,4DCP on M-DF-RGO (experimental conditions: initial chlorophenol concentration $=100 \mathrm{mg} \mathrm{L}^{-1}$, adsorbent dosage $=1.0 \mathrm{~g} \mathrm{~L}^{-1}$, contact time $=12 \mathrm{~h}$ at $298 \mathrm{~K}$ ).

the adsorption since a sufficient number of adsorption sites were available. The adsorption/desorption equilibrium of two types of chlorophenol on M-DF-RGO was obtained within $10 \mathrm{~min}$, and then the adsorption capacities remained almost constant with increasing contact time. In comparison with previously reported adsorbents for the adsorption of chlorophenols ${ }^{49-51}$ the adsorption equilibrium time for the chlorophenols was short due to the single sheet structure of MDF-RGO with good dispersion in water, which enables the adsorbate to be fully in contact with the plane of the adsorbent.

3.2.3 Adsorption isotherms. The adsorption isotherms of 4-CP and 2,4-DCP on the M-DF-RGO hybrid were measured (Fig. 7), and they fitted well with the Freundlich adsorption model:

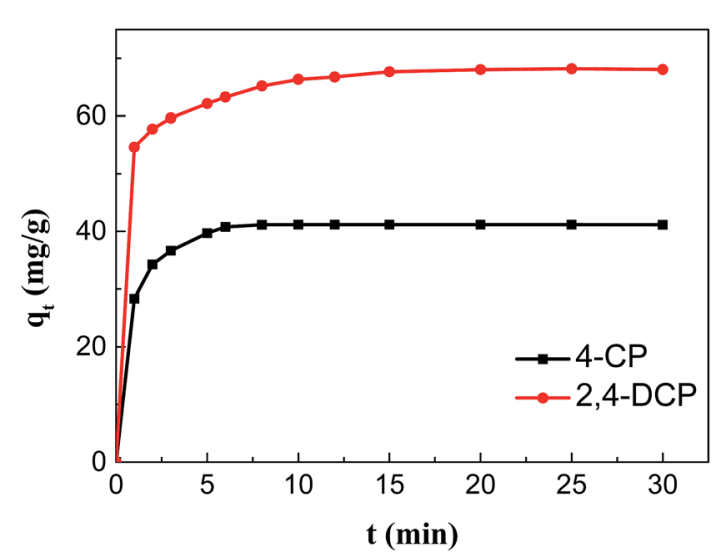

Fig. 6 Adsorption kinetics of chlorophenols on M-DF-RGO (experimental conditions: initial chlorophenol concentration $=100 \mathrm{mg} \mathrm{L}^{-1}$, $\mathrm{pH}=6$ and adsorbent dosage $=1.0 \mathrm{~g} \mathrm{~L}^{-1}$ at $298 \mathrm{~K}$ ). 


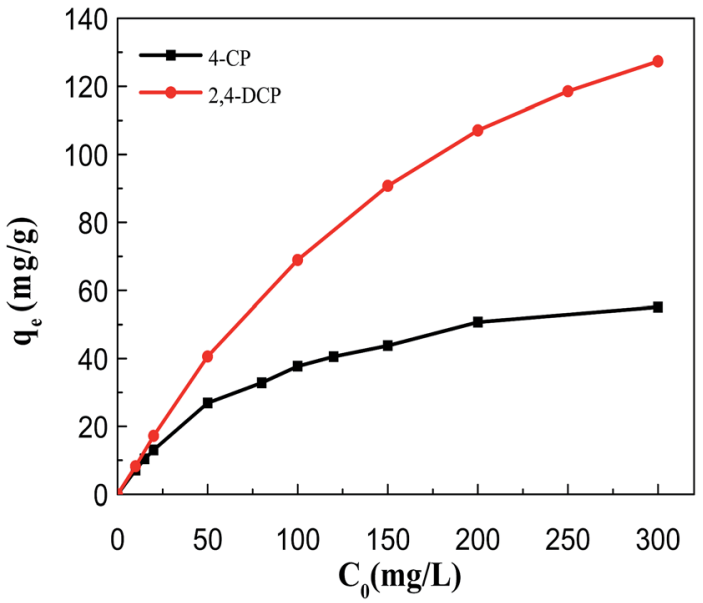

Fig. 7 Adsorption isotherms of 4-CP and 2,4-DCP on M-DF-RGO (experimental conditions: initial chlorophenol concentration $=10-$ $300 \mathrm{mg} \mathrm{L}^{-1}, \mathrm{pH}=6$, adsorbent dosage $=1.0 \mathrm{~g} \mathrm{~L}^{-1}$ and contact time $=$ $30 \mathrm{~min}$ at $298 \mathrm{~K})$. (a)

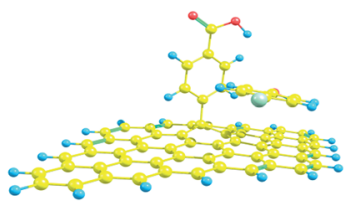

(b)

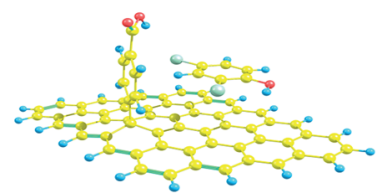

Fig. 8 Optimized structures of the adsorption of (a) 4-CP and (b) 2,4DCP on DF-RGO (carbon atom: yellow; chlorine atom: green; oxygen atom: red; hydrogen atom: blue).

Table 2 Optimized adsorption energies $\left(E_{\mathrm{ad}}\right)$ of 4-CP and 2,4-DCP on DF-RGO

\begin{tabular}{ll}
\hline System & $E_{\text {ad }}\left(\mathrm{kcal} \mathrm{mol}^{-1}\right)$ \\
\hline DF-RGO + 4-CP & 11.61 \\
DF-RGO + 2,4-DCP & 14.56
\end{tabular}

$$
q_{\mathrm{e}}=K_{\mathrm{F}} C_{\mathrm{e}}^{1 / n}
$$

where $K_{\mathrm{F}}\left(\mathrm{L} \mathrm{mg}^{-1}\right), C_{\mathrm{e}}$ is the concentration of chlorophenol at equilibrium ( $\left.\mathrm{mg} \mathrm{L}^{-1}\right)$; and $n$ are the Freundlich constants, which are linked to the relative capacity and adsorption intensity, respectively. The parameters of the Freundlich model for the adsorption of chlorophenols on M-DF-RGO obtained from the experimental data are shown in Table S1. $\dagger$ The stronger interaction between the M-DF-RGO hybrid and the chlorophenols is responsible for the increase of adsorption capacity on M-DF-RGO compared to those of most previously reported adsorbents, ${ }^{\mathbf{5 0 , 5 2 - 5 4}}$ while the inorganic magnetic nanoparticles in M-DF-RGO do not contribute to adsorption.

Some researchers have proved that graphene based materials with $\pi$-electron-rich properties had a good adsorption effect on aromatic compounds through their $\pi$-electroncoupling ability. ${ }^{55,56}$ The reduction of GO with TUD removed

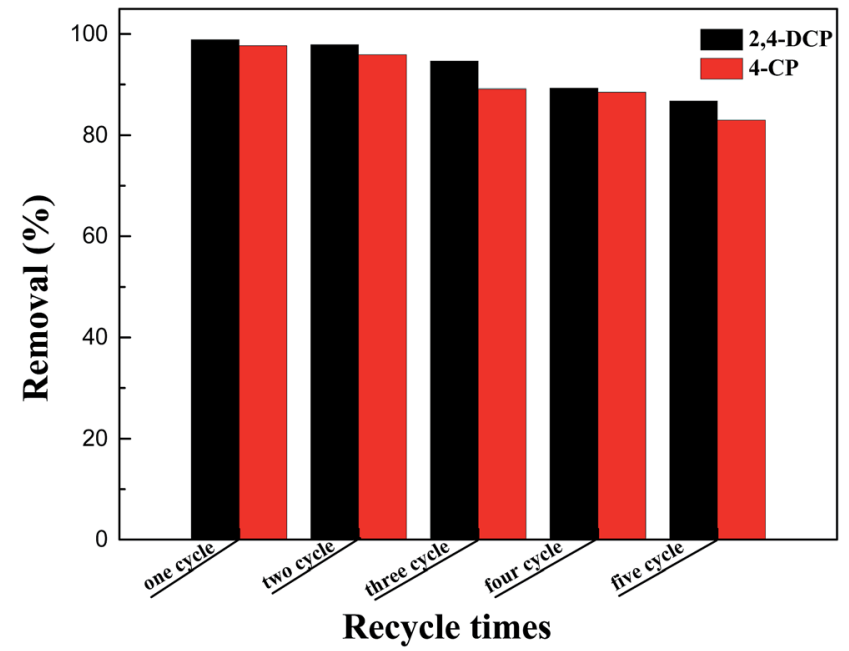

Fig. 9 Recyclability of M-DF-RGO in the removal of chlorophenols (experimental conditions: initial chlorophenol concentration = $100 \mathrm{mg} \mathrm{L}^{-1}, \mathrm{pH}=6$, adsorbent dosage $=1.0 \mathrm{~g} \mathrm{~L}^{-1}$ and contact time $=$ $30 \mathrm{~min}$ at $298 \mathrm{~K}$ ).

some of the functional groups on the plane of GO, and the introduction of the $p$-carboxyphenyl group can restrain the agglomeration of RGO in water. Efforts were made to increase the $\pi-\pi$ interaction between RGO and chlorophenols. Moreover, some remaining oxygen-containing functional groups for DF-RGO could form hydrogen bonds with the hydroxyl groups of phenol. ${ }^{57,58}$

It should also be noted that the adsorption capacities of chlorophenols on M-DF-RGO were remarkably distinguished from each other. The adsorbed amounts of 2,4-DCP with two -Cl on phenol was higher than that of 4-CP with one, suggesting that the number of substituents would significantly influence donor/acceptor strength, ${ }^{55}$ and chlorophenol with more substituted groups had a higher adsorption capacity.

\subsection{DFT calculations}

DFT calculations were applied to investigate the interaction of chlorophenols with DF-RGO. The optimized structures of two chlorophenols on DF-RGO are shown in Fig. 8, and their corresponding adsorption energies $\left(E_{\text {ad }}\right)$ are summarized in Table 2.

As shown in Fig. 8a and b, chlorophenol molecules were parallel to the basal plane of graphene, indicating that the $\pi-\pi$ interaction plays an important role in the removal of chlorophenols. As shown in Table 2, the $E_{\text {ad }}$ value for 2,4-DCP on DFRGO is $14.56 \mathrm{kcal} \mathrm{mol}^{-1}$, which is slightly higher than the $11.61 \mathrm{kcal} \mathrm{mol}^{-1}$ for 4-CP on DF-RGO, indicating that the adsorption of 2,4-DCP on the DF-RGO model surface is more stable and the interaction between DF-RGO and 2,4-DCP is stronger. This might be because the 2,4-DCP molecule has an extra $-\mathrm{Cl}$ group that could strengthen the $\pi-\pi$ interaction between the phenol ring and the adsorbent.

Therefore, based on the results of the theoretical calculations, the higher $E_{\mathrm{ad}}$ value indicated that the DF-RGO had 
a stronger affinity to $2,4-\mathrm{DCP}$ than $4-\mathrm{CP}$, which is in good agreement with the experimental results.

\subsection{Separation and reuse of M-DF-RGO}

Separating the adsorbent from aqueous solution after adsorption was a major procedure in the practical application. From the inset of Fig. 3, it can be seen that M-DF-RGO can be easily separated from aqueous solution under an external magnetic field in a relatively short time, which evidently results from a high load of $\mathrm{Fe}_{3} \mathrm{O}_{4}$ on the surface of M-DF-RGO.

The regeneration and reuse of adsorbents is also important in water treatment based on stringent ecological and economic demands for sustainability. We found that M-DF-RGO can go through multiple rounds of reuse by thorough washing with $0.01 \mathrm{~mol} \mathrm{~L}^{-1} \mathrm{NaOH}$ solution and ethanol, separately. Fig. 9 showed that the adsorption capacities of chlorophenols slightly decreased with increasing times of reuse, and were still over $80 \%$ after reuse in five cycles, suggesting that M-DF-RGO has good reusability.

\section{Conclusion}

A magnetite/diazonium functionalized-reduced graphene oxide (M-DF-RGO) hybrid was successfully prepared. Benefiting from the combined advantages of DF-RGO and $\mathrm{Fe}_{3} \mathrm{O}_{4}$ nanoparticles, the M-DF-RGO composite not only possessed relatively high adsorption efficiency for chlorophenols, but could also be easily separated by an external magnetic field in a fairly short time, which could possibly reduce water treatment expenses. Furthermore, density functional theory calculations indicated that the $\pi-\pi$ interaction played an important role in the removal of chlorophenols. The adsorption capacity of the regenerated M-DF-RGO was still over $80 \%$ after five cycles. All of these results indicated that M-DF-RGO can be utilized as an ideal adsorbent for the simple and rapid removal of phenolic pollutants from aqueous solution.

\section{Conflicts of interest}

There are no conflicts of interest to declare.

\section{Acknowledgements}

This work was supported by the National Natural Science Foundation of China (grant no. 21677087) and National Science and Technology Major Project of China "Environmental Protection Technology Integration and Key Equipment for Tight Reservoir Development" (2016ZX05040-005).

\section{Notes and references}

1 T. Matias, J. Marques, M. J. Quina, L. Gando-Ferreira, A. J. M. Valente, A. Portugal and L. Durães, Colloids Surf., $A, 2015,480,260-269$.

2 Q. Yang, M. Gao and W. Zang, Colloids Surf., A, 2017, 520, 805-816.
3 M. Ahmaruzzaman and S. L. Gayatri, J. Chem. Eng. Data, 2011, 56, 3004-3016.

4 M. H. Dehghani, M. Mostofi, M. Alimohammadi, G. McKay, K. Yetilmezsoy, A. B. Albadarin, B. Heibati, M. AlGhouti, N. M. Mubarak and J. N. Sahu, J. Ind. Eng. Chem., 2016, 35, 63-74.

5 P. Strachowski and M. Bystrzejewski, Colloids Surf., A, 2015, 467, 113-123.

6 J. Fan, J. Zhang, C. Zhang, L. Ren and Q. Shi, Desalination, 2011, 267, 139-146.

7 S. Zhang, X. Zhao, H. Niu, Y. Shi, Y. Cai and G. Jiang, J. Hazard. Mater., 2009, 167, 560-566.

8 Y. Wang, Y. N. Zhang, G. Zhao, H. Tian, H. Shi and T. Zhou, ACS Appl. Mater. Interfaces, 2012, 4, 3965-3972.

9 X. Zhang, A. Li, Z. Jiang and Q. Zhang, J. Hazard. Mater., 2006, 137, 1115-1122.

10 J. S. Valente, F. Tzompantzi, J. Prince, J. G. H. Cortez and R. Gomez, Appl. Catal., B, 2009, 90, 330-338.

11 Z. W. Ming, C. J. Long, P. B. Cai, Z. Q. Xing and B. Zhang, J. Hazard. Mater., 2006, 128, 123-129.

12 G. Liao, W. Zhao, Q. Li, Q. Pang and Z. Xu, Chem. Lett., 2017, 46, 1631-1634.

13 Y. Shen and B. Chen, Environ. Sci. Technol., 2015, 49, 73647372.

14 C. K. Chua, A. Ambrosi and M. Pumera, J. Mater. Chem., 2012, 22, 11054.

15 K. I. Bolotin, K. J. Sikes, Z. Jiang, M. Klima, G. Fudenberg, J. Hone, P. Kim and H. L. Stormer, Solid State Commun., 2008, 146, 351-355.

16 C.-M. Chen, Q. Zhang, M.-G. Yang, C.-H. Huang, Y.-G. Yang and M.-Z. Wang, Carbon, 2012, 50, 3572-3584.

17 L. S. Zhang, W. D. Wang, X. Q. Liang, W. S. Chu, W. G. Song, W. Wang and Z. Y. Wu, Nanoscale, 2011, 3, 2458-2460.

18 V. Georgakilas, M. Otyepka, A. B. Bourlinos, V. Chandra, N. Kim, K. C. Kemp, P. Hobza, R. Zboril and K. S. Kim, Chem. Rev., 2012, 112, 6156-6214.

19 B. Jia and L. Zou, Carbon, 2012, 50, 2315-2321.

20 S. Chatterjee, R. K. Layek and A. K. Nandi, Carbon, 2013, 52, 509-519.

21 X. Huang, X. Qi, F. Boey and H. Zhang, Chem. Soc. Rev., 2012, 41, 666-686.

22 S. Mao, H. Pu and J. Chen, RSC Adv., 2012, 2, 2643.

23 N. Pan, D. Guan, Y. Yang, Z. Huang, R. Wang, Y. Jin and C. Xia, Chem. Eng. J., 2014, 236, 471-479.

24 D. C. Marcano, D. V. Kosynkin, J. M. Berlin, A. Sinitskii, Z. Sun, A. Slesarev, L. B. Alemany, W. Lu and J. M. Tour, ACS Nano, 2010, 4, 4806-4814.

25 J. P. Perdew, K. Burke and M. Ernzerhof, Phys. Rev. Lett., 1996, 77, 3865.

26 W. J. Hehre, R. Ditchfield and J. A. Pople, J. Chem. Phys., 1972, 56, 2257-2261.

27 P. C. Hariharan and J. A. Pople, Theor. Chem. Acc., 1973, 28, 213-222.

28 S. Grimme, J. Antony, S. Ehrlich and H. Krieg, J. Chem. Phys., 2010, 132, 154104.

29 S. F. Boys and F. Bernardi, Mol. Phys., 2006, 19, 553-566.

30 P. A. Denis, ChemPhysChem, 2013, 14, 3271-3277. 
31 P. A. Denis, J. Phys. Chem. C, 2013, 117, 3895-3902.

32 P. A. Denis, Chem. Phys. Lett., 2017, 672, 70-79.

33 Y. Fu, J. Wang, Q. Liu and H. Zeng, Carbon, 2014, 77, 710721.

34 J. Shen, M. Shi, H. Ma, B. Yan, N. Li and M. Ye, Mater. Res. Bull., 2011, 46, 2077-2083.

35 Y. Gao, Y. Li, L. Zhang, H. Huang, J. Hu, S. M. Shah and X. Su, J. Colloid Interface Sci., 2012, 368, 540-546.

36 J. Shen, Y. Hu, M. Shi, N. Li, H. Ma and M. Ye, J. Phys. Chem. C, 2010, 114, 1498-1503.

37 G. Liao, J. Chen, W. Zeng, C. Yu, C. Yi and Z. Xu, J. Phys. Chem. C, 2016, 120, 25935-25944.

38 X. Mei and J. Ouyang, Carbon, 2011, 49, 5389-5397.

39 G. Wei, M. Yan, R. Dong, D. Wang, X. Zhou, J. Chen and J. Hao, Chemistry, 2012, 18, 14708-14716.

40 W. Zhao, M. Fang, F. Wu, H. Wu, L. Wang and G. Chen, J. Mater. Chem., 2010, 20, 5817.

41 J. Li, H. Lin, Z. Yang and J. Li, Carbon, 2011, 49, 3024-3030.

42 Y. Wang, L. Meng, L. Fan, G. Wu, L. Ma, M. Zhao and Y. Huang, Appl. Surf. Sci., 2016, 362, 341-347.

43 N. A. Zubir, C. Yacou, J. Motuzas, X. Zhang and J. C. Diniz da Costa, Sci. Rep., 2014, 4, 4594.

44 A.-L. Morel, S. I. Nikitenko, K. Gionnet, A. Wattiaux, J. LaiKee-Him, C. Labrugere, B. Chevalier, G. Deleris, C. Petibois and A. Brisson, ACS Nano, 2008, 2, 847-856.

45 Y. Zhu, A. L. Higginbotham and J. M. Tour, Chem. Mater., 2009, 21, 5284-5291.
46 X. Yang, X. Zhang, Y. Ma, Y. Huang, Y. Wang and Y. Chen, J. Mater. Chem., 2009, 19, 2710.

47 G. Xie, P. Xi, H. Liu, F. Chen, L. Huang, Y. Shi, F. Hou, Z. Zeng, C. Shao and J. Wang, J. Mater. Chem., 2012, 22, 1033-1039.

48 H. Yan, H. Li, X. Tao, K. Li, H. Yang, A. Li, S. Xiao and R. Cheng, ACS Appl. Mater. Interfaces, 2014, 6, 9871-9880.

49 L. Zhang, B. Zhang, T. Wu, D. Sun and Y. Li, Colloids Surf., A, 2015, 484, 118-129.

50 Ihsanullah, H. A. Asmaly, T. A. Saleh, T. Laoui, V. K. Gupta and M. A. Atieh, J. Mol. Liq., 2015, 206, 176-182.

51 G. C. Chen, X. Q. Shan, Y. S. Wang, B. Wen, Z. G. Pei, Y. N. Xie, T. Liu and J. J. Pignatello, Water Res., 2009, 43, 2409-2418.

52 F. Li and M. J. Rosen, J. Colloid Interface Sci., 2000, 224, 265271.

53 Z. Rawajfih and N. Nsour, J. Colloid Interface Sci., 2006, 298, 39-49.

54 A. Khenifi, B. Zohra, B. Kahina, H. Houari and D. Zoubir, Chem. Eng. J., 2009, 146, 345-354.

55 X. Wang, S. Huang, L. Zhu, X. Tian, S. Li and H. Tang, Carbon, 2014, 69, 101-112.

56 Z. Pei, L. Li, L. Sun, S. Zhang, X.-q. Shan, S. Yang and B. Wen, Carbon, 2013, 51, 156-163.

57 D. Cortés Arriagada, L. Sanhueza and K. Wrighton, Int. J. Quantum Chem., 2013, 113, 1931-1939.

58 Z. Jin, X. Wang, Y. Sun, Y. Ai and X. Wang, Environ. Sci. Technol., 2015, 49, 9168-9175. 